\title{
Conhecimento da equipe de enfermagem de setores críticos na realização e interpretação de eletrocardiograma
}

\author{
Knowledge of the nursing team of critical sectors during the performance and \\ interpretation of an electrocardiogram
}
Conocimiento del equipo de enfermería de sectores críticos en el desempeño e interpretación del electrocardiograma

\begin{abstract}
Darlene Guimarães Ribeiro ${ }^{1}$, Fabiane Frigotto de Barros ${ }^{2}$
${ }^{1}$ Enfermeira. Graduada em Enfermagem pelas Faculdades Pequeno Príncipe. Curitiba - Paraná. ${ }^{2}$ Enfermeira. Mestre em Ensino nas Ciências da Saúde. Docente das Faculdades Pequeno Príncipe. Curitiba - Paraná.
\end{abstract}

\section{RESUMO}

Estudo exploratório-descritivo de abordagem quantitativa, objetivou analisar o conhecimento da equipe de enfermagem dos setores críticos mediante realização e interpretação de eletrocardiograma. Os participantes foram 35 profissionais, entre enfermeiros e técnicos de enfermagem, de setores críticos de um hospital privado de Curitiba. Para a coleta de dados foi utilizado um formulário com vinte questões de múltipla escolha elaborado pela pesquisadora e aplicado através do Google forms ${ }^{\circledR}$. A análise foi estatística descritiva, efetivada por meio do Software Microsoft Excel ${ }^{\circledR}$. Concluiu-se com sua realização que o uso do ECG é imprescindível nos setores críticos e que a equipe de enfermagem possui necessidade de treinamentos

Autor de Correspondência:

*Fabiane Frigotto de Barros . E-mail: fabianefrigottodebarros@gmail.com 
principalmente sobre leitura e interpretação deste exame. Ressalta-se também a importância da educação permanente e a carência literária sobre este tema voltado para a equipe técnica de enfermagem.

Palavras-chave: Cardiologia. Eletrocardiografia. Educação Continuada Permanente. Equipe de Enfermagem .

\section{ABSTRACT}

This is an exploratory-descriptive study with a quantitative approach, aimed to analyze the knowledge of the nursing team in critical sectors during the performance and interpretation of the electrocardiogram. The participants were 35 professionals, including nurses and nursing technicians, from critical sectors of a private hospital in Curitiba. For data collection, a form with twenty multiple-choice questions, prepared by the researcher and applied through Google forms, was used. The analysis was descriptive statistics, performed using Microsoft Excel Software. It was concluded that the use of ECG is essential in critical sectors, and that the nursing team needs training, mainly for reading and interpreting this exam. The importance of continuing education and the lack of literature on this topic aimed at the technical nursing team are also highlighted.

Keywords: Cardiology. Electrocardiography. Education, Continuing Permanent Education. Nursing, Team.

\section{RESUMEN}

Estudio exploratorio descriptivo con enfoque cuantitativo, cuyo objetivo fue analizar el conocimiento del equipo de enfermería en sectores críticos mediante la realización e interpretación del electrocardiograma. Los participantes fueron 35 profesionales, incluidas enfermeras y técnicos de enfermería, de sectores críticos de un hospital privado en Curitiba. Para la recopilación de datos, se utilizó un formulario con veinte preguntas de opción múltiple preparadas por el investigador y aplicadas a través de los formularios de Google. El estudio fue estadístico descriptivo, realizado con el software Microsoft Excel. Se concluyó al darse cuenta de que el uso de ECG es esencial en sectores críticos, y que el equipo de enfermería necesita capacitación principalmente en lectura e interpretación de este examen, también se destaca la importancia de la educación continua y la falta literaria sobre este tema dirigido al equipo técnico de enfermería.

Palabras clave: Cardiología. Electrocardiografía. Educación Continua permanente. 


\section{INTRODUÇÃO}

O processo de urbanização, atrelado ao desenvolvimento tanto da indústria quanto da tecnologia, provocou em diversos povos alterações significativas no estilo de vida, seja no âmbito de alimentação, inadequada, rica em lipídios e alimentos processados, quanto na falta de tempo para prática de atividade física. Essas alterações têm implicado na modificação do perfil epidemiológico da população, uma vez que as Doenças Crônicas Não Transmissíveis (DCNT) estão se sobrepondo às doenças infectocontagiosas ${ }^{1}$.

No Brasil, as DCNTs correspondem a 72\% das causas de óbitos, sendo $31,3 \%$ de doenças do aparelho circulatório, constituindo um problema de saúde de elevada magnitude ${ }^{2}$.

No ano de 2008, de acordo com o Ministério da Saúde, as doenças cardiovasculares (DCV) foram responsáveis por $40,8 \%$ dos óbitos de indivíduos com 60 anos ou mais ${ }^{3}$.

São denominadas DCVs um grupo de doenças do miocárdio e vasos sanguíneos. A OMS estima que no ano de 2030 quase 23,6 milhões de pessoas morrerão de doenças cardiovasculares ${ }^{4}$.

Entre as doenças cardiovasculares mais comuns têm-se os distúrbios no ritmo do miocárdio, as arritmias, que são caracterizados por batimentos rápidos, lentos ou irregulares. Essas alterações podem surgir por anormalidades na gênese e/ou na condução do impulso elétrico. São inúmeros os fatores diretamente relacionados à fisiopatologia desses distúrbios, tais como doenças das artérias coronárias, doenças do músculo cardíaco, doenças valvares, alterações eletrolíticas, doenças infecciosas (doença de chagas, miocardites), doenças infiltrativas (sarcoidose), pós-cirurgia cardíaca ou congênita. Além disso, as arritmias também podem surgir muitas vezes por fatores externos, como cafeína, bebidas alcoólicas, energéticos, cigarro, drogas e estresse. O eletrocardiograma (ECG) é imprescindível no diagnóstico das arritmias 5 .
No Brasil, a estimativa é de 300 a 400 mil casos anuais de infarto agudo do miocárdio, com um óbito a cada cinco a sete casos. O termo síndrome coronariana aguda (SCA) é compatível com isquemia miocárdica aguda, a qual abrange desde a angina instável até o infarto agudo do miocárdio (IAM), seja este com ou sem supradesnivelamento do segmento $\mathrm{ST}^{6,7}$.

O ECG ainda é considerado o padrão ouro para o diagnóstico não invasivo de arritmias cardíacas e isquemia coronariana, podendo ser usado também como método complementar para detecção de alterações estruturais e metabólicas ${ }^{6}$.

Segundo a literatura, a variação no tempo portaECG e no tempo porta-agulha é de 22 a 80 e 140 a 270 minutos, respectivamente. Um estudo realizado na Inglaterra em 2002 relatou o tempo porta-agulha entre cinco e 300 minutos, com redução para cinco a 70 minutos após a introdução de medidas para aperfeiçoar o atendimento com intervenções dos enfermeiros no reconhecimento do infarto ${ }^{8}$.

É de extrema importância que o enfermeiro seja capaz de reconhecer traçados eletrocardiográficos normais e patológicos, considerando o fato de que ele é um dos profissionais da equipe assistencial que permanece continuamente ao lado do paciente ${ }^{6}$.

Para ampliar a continuidade da formação dos profissionais de saúde, garantindo acesso a conhecimento, desenvolvimento de habilidades para prática, atualização e fortalecimento de competências relevantes, a educação permanente tem sido descrita como importante estratégia?

A partir da problematização dos contratempos cotidianos, decorrentes da área de atuação da enfermagem, é possível identificar as necessidades de qualificação, com intuito de provocar mudanças nos modos de agir e produzir serviços de saúde, garantindo desta forma aplicabilidade e relevância dos conteúdos e tecnologias aplicados através de treinamentos e educação permanente ${ }^{10}$. 
Um tempo prolongado para a realização do ECG pode contribuir para a piora clínica nos pacientes com SCA, visto que a demora para a realização do ECG pode resultar em intervenções tardias, impactando, diretamente, nas medidas decisivas para salvar o miocárdio, tais como a administração do trombolítico venoso ou para a realização da angioplastia coronária percutânea ${ }^{11}$.

Dada a importância deste exame e impacto na rotina diária da equipe de enfermagem principalmente em setores críticos, emerge a seguinte questão de pesquisa: "Qual o conhecimento da equipe de enfermagem de setores críticos, no ambiente hospitalar, quanto à realização e interpretação de eletrocardiograma?”.

O objetivo desta pesquisa foi analisar o conhecimento da equipe de enfermagem dos setores críticos mediante realização e interpretação de eletrocardiograma.

\section{MÉTODO}

Trata-se de um estudo exploratório-descritivo, com abordagem quantitativa, realizado mediante formulário aplicado à equipe de enfermagem de um hospital privado de Curitiba referência em cardiologia, no período de setembro a outubro de 2019. A amostra foi composta por 35 formulários, respondidos por enfermeiros e técnicos de enfermagem de setores críticos. Foram incluídos na amostra enfermeiros e técnicos de enfermagem, atuantes no Pronto Atendimento, Unidade Coronariana e Cerebrovascular ou Unidade de Terapia Intensiva, que estavam regularmente inseridos nas escalas de trabalho. Foram excluídos da amostra enfermeiros e técnicos de enfermagem no período de férias e/ou qualquer outro tipo de afastamento.

A pesquisa foi realizada através de formulário fechado com treze questões objetivas de múltipla escolha, acerca do conhecimento da equipe de enfermagem sobre a elaboração e interpretação de ECG, elaborado pela pesquisadora principal e aplicado através da plataforma Google forms ${ }^{\circledR}$. Cada uma das questões de pesquisa será apresentada como uma variável nos resultados deste artigo. $\mathrm{O}$ envio para as equipes de enfermagem foi realizado pelos gestores dos setores citados, através do aplicativo de mensagens Whatsapp $^{\circledR}$, os quais possuem o contato telefônico das equipes e da pesquisadora, para mediação. Ressalta-se que o Google forms ${ }^{\circledR}$ é uma ferramenta de propriedade da Google ${ }^{\circledR}$, com permissão para livre utilização pelo público de interesse.

A análise dos dados foi estatística simples, por meio de gráficos gerados pelo software Microsoft Excel ${ }^{\circledR}$.

Esta pesquisa foi aprovada pelo Comitê de Ética em Pesquisa, mediante parecer $n^{\circ}$ 3.588.985. A coleta de dados foi iniciada somente após esta aprovação. Para a realização desta pesquisa foram respeitadas as Diretrizes e Normas Regulamentadoras de Pesquisas envolvendo seres humanos, emanadas da Resolução no 466, de 2012 do Conselho Nacional de Saúde ${ }^{12}$.

\section{RESULTADOS}

A análise dos dados se deu por meio de estatística simples e os resultados serão apresentados por meio de tabelas. A tabela apresentada a seguir demonstra o perfil dos participantes da pesquisa, quanto a categoria profissional e setor de atuação.

Como evidenciado na tabela 1, dos 35 formulários respondidos pelos participantes que compuseram a amostra da pesquisa, 10 participantes (29\%) são enfermeiros, e 25 participantes (71\%), são técnicos de enfermagem.

Quanto ao setor de trabalho dos participantes da pesquisa, 5 participantes (14\%) trabalham no Pronto Atendimento, 15 (43\%) trabalham na Unidade de Terapia Intensiva, e 15 (43\%) trabalham na Unidade Coronariana. Foi evidenciada no estudo a maior taxa de participação por parte dos setores fechados, ficando o Pronto Atendimento com a menor taxa de participação. 
Tabela 1 - Perfil dos participantes da pesquisa

\begin{tabular}{l|c|c}
\hline \multicolumn{3}{c}{ PROFISSÃO } \\
\hline \multicolumn{1}{c}{ n } & \% \\
\hline Enfermeiro & 10 & $29 \%$ \\
\hline Técnicos de enfermagem & 25 & $71 \%$ \\
\hline \multicolumn{1}{c}{ SETOR DE ATUAÇÃO } \\
\hline Unidade de Terapia Intensiva & $\mathbf{n}$ & $\mathbf{\%}$ \\
\hline Unidade Coronariana & 15 & $43 \%$ \\
\hline Pronto Atendimento & 15 & $43 \%$ \\
\hline
\end{tabular}

Fonte: Dados da pesquisa, 2019.

Os dados levantados em relação aos conhecimentos para o exame e sua realização serão apresentados na dos participantes quanto a preparação do paciente tabela 2.

Tabela 2 - Conhecimento sobre o preparo e a realização do exame.

\begin{tabular}{|c|c|c|}
\hline \multicolumn{3}{|c|}{ Orientações teóricas sobre ECG durante a formação profissional } \\
\hline & $\mathrm{n}$ & $\%$ \\
\hline Consideram que receberam adequadamente & 26 & $74 \%$ \\
\hline Não consideram ter recebido adequadamente & 5 & $14 \%$ \\
\hline Talvez receberam as orientações & 4 & $12 \%$ \\
\hline \multicolumn{3}{|c|}{ Conhecimento quanto às orientações pré-exame que devem ser realizadas ao paciente } \\
\hline & $\mathrm{n}$ & $\%$ \\
\hline Conhecem totalmente & 23 & $66 \%$ \\
\hline Conhecem parcialmente & 12 & $34 \%$ \\
\hline Desconhecem & 0 & $0 \%$ \\
\hline \multicolumn{3}{|c|}{ Conhecimento quanto ao posicionamento anatômico dos eletrodos } \\
\hline & $\mathrm{n}$ & $\%$ \\
\hline Conhecem totalmente & 29 & $83 \%$ \\
\hline Conhecem parcialmente & 6 & $17 \%$ \\
\hline Desconhecem & 0 & $0 \%$ \\
\hline \multicolumn{3}{|c|}{ Conhecimento quanto ao posicionamento dos cabos e às respectivas derivações } \\
\hline & $\mathrm{n}$ & $\%$ \\
\hline Conhecem totalmente & 25 & $72 \%$ \\
\hline Conhecem parcialmente & 9 & $26 \%$ \\
\hline Desconhecem & 1 & $2 \%$ \\
\hline
\end{tabular}


Quanto à formação, 26 participantes (74\%) responderam que receberam orientações teóricas sobre o ECG, $4(12 \%)$ responderam que talvez tenham recebido essas orientações, e 5 participantes (14\%) alegaram não receber nenhuma orientação teórica sobre ECG seja na graduação ou curso técnico.

Sobre as orientações que devem ser feitas aos pacientes antes da realização do exame, dos 10 enfermeiros participantes, 6 (60\%) afirmaram conhecer totalmente estas orientações, e 4 (40\%) dos enfermeiros responderam conhecer parcialmente.

Quanto às orientações pré-exame aos pacientes, 68\% dos técnicos de enfermagem responderam conhecêlas totalmente, ou seja, 17 participantes. Os outros 8 participantes, $32 \%$, responderam que conhecem parcialmente estas orientações.

A respeito do posicionamento dos eletrodos em relação à anatomia, dos 35 participantes da pesquisa, 29 (83\%) afirmaram conhecer totalmente, e os outros $6(17 \%)$ responderam que conhecem parcialmente esse posicionamento.

Dos enfermeiros que participaram da pesquisa, 5 (50\%) afirmaram que sabem diferenciar um ECG dentro da normalidade de um ECG com alterações, 4 enfermeiros (40\%) responderam que talvez conseguissem identificar um ECG alterado, e 1 (10\%) afirmou não conhecer o suficiente para diferenciar um traçado eletrocardiográfico alterado de um dentro dos padrões de normalidade.

Sobre o mesmo questionamento aos técnicos de enfermagem - de conseguir identificar um ECG com alterações -, 28\% responderam que sim, $44 \%$ alegaram que talvez conseguissem, e 28\% declararam não conseguir fazer essa diferenciação.

Sobre conhecimento suficiente para identificar um ECG de paciente com IAM, 23\% da equipe de enfermagem afirmou que sim, tem o conhecimento para identificar no ECG um IAM, 23\% responderam que não, e 54\%, a maioria, alegaram que talvez identificassem um IAM por este exame.

Tão importante quanto a realização do exame propriamente dita, é a capacidade da equipe de enfermagem de interpretar os resultados obtidos. Os dados sobre o conhecimento dos participantes acerca da interpretação de ECG são apresentados na tabela 3.

No âmbito ainda de interpretação, 10\% dos enfermeiros afirmaram conhecer totalmente sobre a correlação dos achados eletrocardiográficos e qual parede ventricular foi acometida, 50\% conhecem parcialmente, e $40 \%$ desconhecem a correlação.

A tabela 4 demonstra a percepção dos participantes da pesquisa acerca da importância do conhecimento sobre a realização e interpretação de ECG para a segurança do paciente e a importância da implementação de estratégias de educação permanente sobre este exame.

No âmbito de agilidade de atendimento mediante a identificação precoce de alterações no ECG, baseado em conhecimento científico, 33 participantes (94\%) responderam que sim, que este conhecimento na prática torna o atendimento mais rápido melhorando o desfecho do quadro clínico do paciente, e 2 participantes (6\%) afirmaram que não, este conhecimento não muda a agilidade na prática.

Sobre a importância do conhecimento da realização e interpretação do ECG, 34 participantes (97\%) concordam totalmente que é de suma importância este tema, apenas 1 participante (3\%) concorda parcialmente. 
Tabela 3 - Conhecimento sobre a interpretação do ECG.

\section{Competência para diferenciar um ECG dentro da normalidade de um ECG com alterações}

\begin{tabular}{l|c|c} 
& $\mathbf{n}$ & $\mathbf{\%}$ \\
\hline Sabem diferenciar & 12 & $35 \%$ \\
\hline Não sabem diferenciar & 8 & $24 \%$ \\
\hline Talvez saibam diferenciar & 15 & $41 \%$ \\
\hline
\end{tabular}

Competência para diferenciar um ECG compatível com um Infarto Agudo do Miocárdio (IAM) de um ECG com ritmo sinusal

\begin{tabular}{l|c|c}
\hline & $\mathbf{n}$ & $\%$ \\
\hline Sabem diferenciar & 8 & $24 \%$ \\
\hline Não sabem diferenciar & 8 & $24 \%$ \\
\hline Talvez saibam diferenciar & 19 & $52 \%$ \\
\hline
\end{tabular}

Conhecimento sobre a leitura da frequência e ritmo cardíaco pelo ECG

\begin{tabular}{l|c|c}
\hline & $\mathbf{n}$ & $\%$ \\
\hline Conhecem totalmente & 5 & $13 \%$ \\
\hline Conhecem parcialmente & 25 & $74 \%$ \\
\hline Desconhecem & 5 & $13 \%$ \\
\hline
\end{tabular}

\section{Conhecimento sobre onda P, complexo QRS e onda T}

\begin{tabular}{l|c|c}
\hline & $\mathbf{n}$ & $\%$ \\
\hline Conhecem totalmente & 7 & $20 \%$ \\
\hline Conhecem parcialmente & 22 & $65 \%$ \\
\hline Desconhecem & 6 & $15 \%$ \\
\hline
\end{tabular}

Conhecimento sobre eletrofisiologia cardíaca e seu impacto nas ondas do ECG

\begin{tabular}{l|c|c}
\hline & $\mathbf{n}$ & $\%$ \\
\hline Conhecem totalmente & 1 & $1 \%$ \\
\hline Conhecem parcialmente & 24 & $70 \%$ \\
\hline Desconhecem & 10 & $29 \%$ \\
\hline
\end{tabular}

Conhecimento quanto à correlação de achados eletrocardiográficos com a parede ventricular acometida

\begin{tabular}{l|c|c}
\hline & $\mathbf{n}$ & $\%$ \\
\hline Conhecem totalmente & 1 & $2 \%$ \\
\hline Conhecem parcialmente & 14 & $40 \%$ \\
\hline Desconhecem & 20 & $58 \%$ \\
\hline
\end{tabular}


Tabela 4 - Percepção dos profissionais sobre a importância do conhecimento sobre ECG e da educação permanente para segurança do paciente.

\begin{tabular}{|c|c|c|}
\hline \multicolumn{2}{|c|}{ Percepção sobre a importância do conhecimento sobre ECG para a segurança do paciente } & $\%$ \\
\hline Concordam totalmente & 34 & $98 \%$ \\
\hline Concordam parcialmente & 1 & $2 \%$ \\
\hline Discordam & 0 & $0 \%$ \\
\hline \multicolumn{3}{|c|}{ Percepção sobre a importância de ações de educação permanente na realização e interpretação de ECG } \\
\hline & $\mathbf{n}$ & $\%$ \\
\hline Acreditam na importância & 32 & $92 \%$ \\
\hline Não acreditam na importância & 3 & $8 \%$ \\
\hline \multicolumn{3}{|c|}{$\begin{array}{l}\text { Percepção sobre a relação entre o conhecimento do ECG e a agilidade e segurança no } \\
\text { atendimento do paciente }\end{array}$} \\
\hline & $\mathrm{n}$ & $\%$ \\
\hline Concordam totalmente & 33 & $95 \%$ \\
\hline Concordam parcialmente & 2 & $5 \%$ \\
\hline Discordam & 0 & $0 \%$ \\
\hline
\end{tabular}

Fonte: Dados da pesquisa, 2019.

\section{DISCUSSÃo}

Em estudo realizado em Recife, $48,9 \%$ dos enfermeiros responderam que o momento de aprendizagem da técnica para a realização do ECG ocorreu durante a graduação, $42,6 \%$ durante a prática assistencial, e $8,5 \%$ em outras ocasiões, como na formação técnica ou cursos de especialização ${ }^{13}$. O estudo citado corrobora com os dados encontrados na pesquisa, já que os participantes que mais relataram conhecimento a respeito de ECG foram os enfermeiros.

A construção do conhecimento sobre ECG apresenta certa heterogeneidade, tendo por diversas vezes, até mesmo nos livros, foco na física, fazendo com que os estudantes de enfermagem e medicina não sintam atração pelo tema, provavelmente porque sua escolha profissional está distante das ciências exatas ${ }^{14}$.
Erros técnicos na realização do ECG podem levar a erros significantes no traçado eletrocardiográfico. Dentre os fatores que podem prejudicar o exame, encontram-se a movimentação excessiva do paciente, conformação do tórax, calibração inadequada, interferências eletromagnéticas e posicionamento errôneo de eletrodos. O desconhecimento de alguns pontos na realização do exame evidenciam lacunas na formação dos enfermeiros e que estão delegando funções à equipe técnica, necessitando de maior embasamento científico em suas práticas profissionais ${ }^{13}$.

Desta forma, é essencial a implementação de estratégias que ampliem os conhecimentos de técnicos de enfermagem sobre o ECG, já que são eles os profissionais da linha de frente da assistência. 
Para a obtenção de um traçado satisfatório e a diminuição da margem de erro e falsos diagnósticos, devem ser seguidas certas especificações: paciente posicionado em decúbito dorsal e imóvel, verificar higiene da pele onde serão aplicados os eletrodos, tricotomia se necessário, evitar uso excessivo de pasta de contato e posicionamento correto dos eletrodos ${ }^{13}$. Todos os cuidados que, em grande parte das vezes, são realizados por técnicos de enfermagem.

Um estudo retrospectivo realizado em hospital universitário sobre segurança do paciente, mostrou prevalência de $82 \%$ de incidentes sem dano. Entre os vários tipos de incidentes identificados, obteve destaque as falhas durante procedimentos técnicos, omissão de cuidados e incidentes relacionados à estrutura organizacional como manutenção de equipamentos. Essas falhas podem levar ao aumento no tempo de atendimento e, consequentemente, pior prognóstico para o paciente ${ }^{15}$.

Um estudo realizado na Inglaterra com médicos e enfermeiros que prestavam atendimento de emergência a pacientes cardíacos internados, mostrou notável variação na colocação dos eletrodos precordiais, com baixo índice de acertos ${ }^{13}$.

Em estudo realizado no Rio Grande do Sul, Brasil, com 24 enfermeiros, apenas um terço deles demonstrou conhecimento correto sobre as derivações utilizadas no $\mathrm{ECG}^{16}$.

O enfermeiro é um dos principais profissionais da equipe assistencial e que permanece em contato direto com o paciente. É de extrema importância que ele tenha capacidade para diferenciar traçados eletrocardiográficos normais de traçados patológicos ${ }^{6}$.

A rápida identificação de alterações no ECG permite a tomada imediata de condutas, já que em curto prazo o IAMCSSST (por extenso antes da sigla) apresenta elevada taxa de mortalidade, tendo um prognóstico pior que o IAMSSST ${ }^{17}$.

Em estudo feito na Suécia, cuja finalidade foi descrever as habilidades de interpretação de ECG pelos enfermeiros de um serviço móvel de urgência, foi identificado que, mesmo aqueles enfermeiros com experiência assistencial em unidades coronarianas, apresentaram $46 \%$ de acertos em média na análise de traçados eletrocardiográficos com supradesnivelamento de segmento ST. Outro estudo, feito com enfermeiros norte-americanos, revelou percentual ainda menor de acertos (20\%) em relação à interpretação de traçados relacionados ao IAM $^{6}$.

O conhecimento do enfermeiro dará subsídios para a interpretação de alterações eletrocardiográficas e clínicas que o paciente possa apresentar, possibilitando a adoção de intervenções imediatas ${ }^{6}$.

Um estudo feito na Carolina do Norte (EUA), mostrou que quanto mais rápida a reperfusão miocárdica, melhor é a função cardíaca e menor chance de reinfarto. Em contrapartida, pacientes que demoraram mais para realizar a reperfusão tiveram uma maior taxa de mortalidade após 30 dias, sendo isto impactado diretamente pelo tempo de atraso no tratamento de pacientes com $\mathrm{IAM}^{18}$.

A morbimortalidade do paciente com IAM é tempo dependente, seja a opção de tratamento a intervenção coronária percutânea ou terapia trombolítica. Quanto mais tempo este paciente aguardar atendimento, pior será seu prognóstico ${ }^{19}$.

$\mathrm{Na}$ ocorrência de troca de cabos, $66 \%$ da equipe de enfermagem que compôs a amostra desta pesquisa afirmou saber identificar, 20\% respondeu que talvez identifique, e $14 \%$ não sabem identificar caso aconteça.

De acordo com a literatura, as trocas dos cabos nos membros podem causar inversão das ondas e o posicionamento em altura elevada do tórax pode criar ondas que mimetizam o IAM132.

Dos 10 enfermeiros participantes da pesquisa, $80 \%$ conhecem parcialmente sobre leitura de frequência e ritmo cardíaco através do ECG, 10\% conhecem 
totalmente e $10 \%$ desconhecem.

É fundamental instrumentalizar os profissionais na interpretação de anormalidades de ritmo, da frequência e da atividade elétrica cardíaca. É enfatizada pela literatura a necessidade de um programa de educação sistemático sobre $\mathrm{ECG}^{16}$.

Um estudo revela que os enfermeiros de unidades críticas sentem-se mais seguros para interpretar ECG do que os das unidades não críticas ${ }^{6}$.

Quanto à equipe, 68\% dos técnicos de enfermagem responderam que conhecem parcialmente sobre leitura de frequência e ritmo cardíaco através de ECG, 16\% afirmaram conhecer totalmente e 16\% desconhecem esta possibilidade sem aprimoramento teórico prático.

Existe uma carência na literatura de estudos direcionados ao técnico de enfermagem quanto à interpretação básica de eletrocardiograma.

A respeito do conhecimento sobre ondas $\mathrm{P}, \mathrm{T}$ e complexo QRS na interpretação do eletrocardiograma, $80 \%$ dos enfermeiros têm parcialmente este conhecimento, e 20\% afirmaram conhecer totalmente estes pontos na interpretação do ECG.

Sendo o enfermeiro o profissional de saúde que permanece na assistência direta ao paciente, ele deve ter conhecimento suficiente para identificar arritmias, prevenindo danos por funcionamento inadequado do miocárdio. Neste âmbito, faz-se necessário que o enfermeiro conheça e compreenda o formato das ondas, o tempo do intervalo entre elas, bem como a frequência cardíaca, sendo de suma importância para o reconhecimento de arritmias, a fim de promover assistência imediata adequada ${ }^{11}$.

Quanto à eletrofisiologia cardíaca e o seu impacto no traçado eletrocardiográfico, $90 \%$ dos enfermeiros têm este conhecimento parcial, e 10\% apenas alegam conhecer totalmente esta relação eletrofisiologia e ECG.
O estudo de 2018 corrobora que o conhecimento da fisiologia cardiovascular é primordial para o enfermeiro, permitindo relacionar as arritmias ao sistema de formação ${ }^{16}$.

Tanto o conhecimento da sintomatologia quanto a correta interpretação de exames são de extrema importância para a assertividade e sucesso no tratamento, já que $52 \%$ dos pacientes com IAMCSST possuem acometimento de parede anterior, o que confere pior prognóstico se comparado ao acometimento de outras paredes cardíacas ${ }^{15}$.

Há diversos estudos na literatura que avaliaram a habilidade de profissionais médicos para interpretar alterações eletrocardiográficas, principalmente aquelas relacionadas à isquemia. Entretanto, é escasso o número de trabalhos que avaliam o conhecimento de enfermeiros quanto à interpretação de ECG ${ }^{6}$.

Se o enfermeiro tiver conhecimento para a rápida identificação e interpretação das alterações apresentadas no ECG de 12 derivações, isto permite a antecipação de eventos potencialmente fatais ao paciente ${ }^{6}$.

O estudo PULSE evidenciou que após um programa interativo de educação com ênfase na análise eletrocardiográfica, aumentou de $82 \%$ para $97 \%$ a acurácia de enfermeiros na interpretação de arritmias cardíacas ${ }^{16}$.

Os resultados desta pesquisa também apontam para a importância da implementação de ações de educação permanente sobre ECG para a agilidade e segurança no atendimento ao paciente.

Resultados de um estudo realizado em Porto Alegre (RS), destacaram a educação permanente em saúde como nova ferramenta no que tange ao saber e ao fazer em enfermagem, repercutindo na segurança e qualidade da assistência prestada aos pacientes ${ }^{9}$.

$\mathrm{Na}$ área da enfermagem, é necessário que haja periodicamente um programa de educação permanente voltado ao desenvolvimento do profissional no contexto teórico e prático em $\mathrm{ECG}^{16}$. 
Em contrapartida, um estudo transversal feito em setores críticos de Hospital escola de Recife mostrou lacunas de conhecimento chamando atenção para a importância de intervenções educativas, no que tange à atuação da educação permanente nesses setores de alta complexidade, visando atendimento de qualidade ${ }^{13}$.

A capacitação deve ser realizada de maneira individualizada, baseada nas necessidades, especificidades da área de atuação e grau de conhecimento prévio do profissional ${ }^{16}$.

\section{CONCLUSÕES}

Concluiu-se, através deste estudo, que no ambiente hospitalar, principalmente em setores críticos, o ECG é amplamente utilizado, seja para identificação de sintomas inespecíficos, para prevenção da deterioração clínica ou fechamento de determinados diagnósticos.

Os resultados revelam o perfil de conhecimento da equipe de enfermagem acerca da realização e interpretação do eletrocardiograma, respondendo a questão de pesquisa.

Os objetivos do estudo foram alcançados, ficando evidente que a maior porcentagem de respostas positivas sobre o conhecimento tanto de técnicos quanto de enfermeiros está no momento de preparação do exame, sendo identificado lacunas no que tange à interpretação do ECG.

A educação permanente é demonstrada nos resultados deste estudo como uma importante estratégia, que deve ser conduzida de acordo com as dificuldades e conhecimento prévio dos profissionais, já que o tema ECG é desafiador e possui especificidades mais complexas do que outros treinamentos.

Como limitações desta pesquisa pode-se citar o recorte temporal e amostra proveniente de uma única instituição.
O benefício deste estudo se dá por meio do levantamento de dados resultantes do inquérito sobre a percepção da própria equipe de enfermagem quanto aos seus conhecimentos sobre ECG, podendo subsidiar futuros treinamentos pautados nas necessidades da equipe e nos princípios da aprendizagem significativa.

Verificou-se um déficit na literatura quanto a estudos direcionados à equipe técnica de enfermagem voltados ao tema, tanto no momento de preparação quanto na leitura do eletrocardiograma. Assim, recomenda-se que mais pesquisas direcionadas a este profissional sejam realizadas nesta área.

\section{REFERÊNCIAS}

1. Silva LN, Karino ME, Martins JT, Galdino MSQ, Scholze AR, Ribas JJ. Perfil epidemiológico e clínico de pacientes com síndrome coronariana aguda. Rev Enferm UFPE. 2018;12(2):379-85.

2. Maier GSO, Martins EAP. Assistência ao paciente com síndrome coronariana aguda segundo indicadores de qualidade. Rev Bras Enferm. 2016;69(3):710-7.

3. Piuvezam G, Medeiros WR, Costa AV, Emerenciano FF, Santos RC, Seabra DS. Mortalidade em Idosos por Doenças Cardiovasculares: Análise Comparativa de Dois Quinquênios. Arq Bras Cardiol. 2015 Oct; 105(4): 371-380.

4. Radovanovic CAT, Santos LA, Carvalho MDB, Marcon SS. Hipertensão arterial e outros fatores de risco associados às doenças cardiovasculares em adultos. Rev Latino-Am Enfermagem. 2014 Aug; 22(4): 547-553.

5. Reis HJL, Guimarães HP, Zazula AD, Vasque RG, Lopes RD. ECG: manual prático de eletrocardiograma. 1. ed. São Paulo: Atheneu; 2013.

6. Santos ES, Pires EC, Silva JT, Sallai VS, Bezerra DG, Ferretti-Rebustini REL. Habilidade dos enfermeiros na interpretação do eletrocardiograma de 12 derivações. Rev Baiana Enferm. 2017; 31(1):e16581.

7. Góis AFT, Teixeira de Demuner MS, Bichuetti DB, Silva Junior M. Emergências Médicas. Rio de Janeiro: Atheneu; 2017 
8. Guimarães DBO, Rodrigues TS, Oliveira SCM, Avelino FVSD. Tempo porta eletrocardiograma em pacientes com dor torácica na emergência. Rev Enferm. UFPE. 2018; 12(4):1027-36.

9. Jesus MC, Silva VA, Mota RS, Costa JCB, Mendes AS, Oliveira MJ. Repercussões da educação permanente nas práticas assistenciais dos profissionais de enfermagem. Rev Baiana Enferm. 2019; 33: e2755.

10. Adamy EK, Zocche DAA, Vendruscolo C, Metelski FK, Argenta C, Valentini JS. Tecendo a educação permanente em saúde no contexto hospitalar: relato de experiência. Rev Enferm Centro-Oeste Mineiro. 2018; 7:e1615.

11. Andrade KBS, Pinheiro APB, Bessa ATT, Paes GO, Stipp MAC. Avaliação do tempo de espera do eletrocardiograma inicial em pacientes com síndrome coronariana aguda. Rev Enferm UERJ. 2015;23 (4):443-448.

12. Brasil. Conselho Nacional de Saúde. Resolução 466 de 12 de dezembro de 2012. Brasília (DF): Diário Oficial da União. 2012.

13. Fernandes LS, Lira MCLS, França VV, Valois AA, Valença MP. Conhecimento teórico-prático de enfermeiras sobre eletrocardiograma. Rev Baiana Enferm. 2015;29 (2): 98-105

14. Barros MNDS, Silva MCA, Oliveira Neto NR, Escarião AG, Albuquerque ALT. Nova metodologia de ensino do ECG: desmistificando a Teoria na Prática - Ensino prático do ECG. Rev Bras Educação Médica. 2016;40(4):751-756.

15. Silva RB, Castro CM, Iser BPM, Castilho LJC. Perfil dos pacientes com síndromes coronarianas agudas em um hospital da Região Sul do Brasil. Rev Soc Bras. 2016; 14(1):33-7.

16. Saffi MAL, Bonfada MS. Conhecimento de enfermeiros no manejo e interpretação do eletrocardiograma. Rev Baiana Enferm. 2018;32: e26004.

17. Santos EB, Bianco HT. Atualizações em doenças cardíacas isquêmica aguda e crônica. Rev Soc Bras Clin Med. 2018;16 (1):52-58.
18. Pinto LLN, Correa AR, Donoso MTV, Matos SS, Manzo BF. Estratégias para reduzir tempo porta-balão nos pacientes com infarto agudo do miocárdio. Rev Min Enferm. 2016; 20:e954.

19. Sant'Anna RT, Miglioransa MH, Leiria TLL. Infarto do miocárdio: devemos transferir o paciente com IAM? Rev Soc Cardiol Rio Grande do Sul. 2007;11:1-6. 